\title{
A preliminary study on synchronous simulation for clinical teaching and learning among medical under-graduate students
}

\author{
Ikram, M.A., Subramaniam, T., Ramasamy, S. \\ International Medical University, Malaysia
}

\section{Background:}

The spread of COVID-19 has forced many countries including Malaysia to implement lockdown to restrict the spread of the virus. This has resulted in a transition from on-campus learning to exclusively online learning. The modality of the synchronous session allows the learners to interact with the instructor and obtain instant feedback. The objective of this study was to evaluate the effectiveness of synchronous simulation among medical students in clinical years in the absence of hospital-based clinical teaching and learning.

\section{Method:}

This is a prospective observational study involving 23 clinical year students, conducted in the clinical skills and simulation lab at International Medical University. The conceptual framework of the Synchronous Simulation Learning Cycle was grounded on Kolb's Experiential Learning Cycle theoretical model (1984). Scenarios for each session were built around clinical skills targeting specific learning outcomes. Synchronous debriefing followed each simulated session allowing active participation by all students. The simulation session was evaluated using a 5-point Likert Scale. Focus group discussion was conducted among 54 students representing online participants to obtain feedback on their learning experience. Data and Statistical Analysis: A descriptive statistic was conducted.

\section{Results:}

Amongst the highest mean scores are 4.093 (0.734), 4.057(0.811), 3.925(1.007), 3.852(0.898) respectively for items referring to team communication, debriefing, timetabling, and transfer of clinical skills to the actual environment. Results from the focus group discussion revealed that; simulated sessions assisted in the application of knowledge by observing their peers; students prefer the repetitive process of observing and performing than observing per se; observing the performance of peers from the same cohort was preferred, and critique on technical logistics.

\section{Conclusion:}

The synchronous simulation sessions were well received by the students. It is believed that this will be a novel teaching modality to adopt even after the passing of the pandemic.

Keywords: Synchronous simulation, Clinical Teaching 